\title{
Erratum: Elasticity bounds from effective field theory [Phys. Rev. D 100, 065015 (2019)]
}

\author{
Lasma Alberte* \\ Theoretical Physics, Blackett Laboratory, Imperial College, London, SW7 2AZ, United Kingdom \\ Matteo Baggioli ${ }^{\dagger}$ \\ Instituto de Fisica Teorica UAM/CSIC, c/Nicolas Cabrera 13-15, Universidad Autonoma de Madrid, \\ Cantoblanco, 28049 Madrid, Spain
}

Víctor Cáncer Castillo ${ }^{\ddagger}$ and Oriol Pujolàs $\odot^{\S}$

Institut de Física d'Altes Energies (IFAE), The Barcelona Institute of Science and Technology (BIST)

Campus UAB, 08193 Bellaterra, Barcelona, Spain

(Received 19 June 2020; published 16 September 2020)

\begin{abstract}
We extend the stability analysis of the configurations under finite strain using Effective Field Theory (EFT) methods done in [L. Alberte, M. Baggioli, V. C. Castillo, and O. Pujolas, Phys. Rev. D 100, 065015 (2019)]. At finite strain the medium is anisotropic and so fluctuations in different directions behave differently. We recompute the bounds by including the instabilities that may occur in all possible directions. Qualitatively, the main conclusions of [Phys. Rev. D 100, 065015 (2019)] remain unchanged: Nontrivial bounds and correlations between different nonlinear observables can be obtained using EFT methods. Quantitatively, the bounds become more stringent—especially so for materials with power-law stress strain curves $\left(\sigma \sim \varepsilon^{\nu}\right)$ characterized with a small exponent $\nu$.
\end{abstract}

DOI: 10.1103/PhysRevD.102.069901

In Appendix A of [1], we computed the effective action for the phonon fluctuations $\pi^{I}$ [see Eqs. (A2) and (A3)]. We have decomposed our fluctuations into transverse and longitudinal with respect to the wave-vector $\vec{k}$ rotated along the strain deformation of the equilibrium configuration. However, beyond linear level, for large strains, our background is not isotropic because of the presence of an external strain configuration $\phi_{\text {str }}^{I}$ which explicitly breaks the spatial SO(2) symmetry. Therefore, our original assumption that the field perturbations can only be made to depend on one of the spatial directions, i.e., that $\pi_{L / T}=\pi_{L / T}(t, x)$ appears unjustified and needs to be corrected. The nonlinear strain-dependent speed of propagation of the phonon fluctuations depends crucially on the angle of propagation $\theta$, which in [1] was considered to be only $\theta=0$ (longitudinal modes) and $\theta=\pi / 2$ (transverse modes). In this erratum, we correct our previous results and we generalize them to a generic angle of propagation $\theta$ introduced as $\pi^{I}=a^{I} e^{-i \omega t+i k x \sin \theta+i k y \cos \theta}$. The main results of [1] remain unchanged.

The complete expression for the speeds $c_{i}^{2}(\theta, \varepsilon)$ is tedious but straightforward to compute from the quadratic action. The angle dependence disappears in the linear limit $c_{i}^{2}(\theta, \varepsilon \rightarrow 0)$ since the background becomes isotropic again.

Using the new expressions for the velocity and scanning all the angles $\theta$ we require that the same consistency requirements are satisfied in all directions: no ghosts, no gradient instabilities and no superluminal modes. In Figs. 1-3 we find the maximum strain applicable $\varepsilon_{\max }$. In Fig. 1 we analyze the simplest of our benchmark models, $V(X, Z)=$ $\rho_{\mathrm{eq}} X^{A} Z^{\frac{B-A}{2}}$, while in Figs. 2 and 3 we work in the extended model with $V(X, Z)$ given in Eq. (24) in [1] (to be compared with Figs. 4-6 in [1]). The main difference that must be pointed out is that now we do not have anymore a region with large $\varepsilon_{\max }$ close to $A=0$. Thus there are no hyperelastic materials with small shear exponent $\left(\nu_{\text {shear }}=2 A\right)$ anymore. Instead, the hyperelastic region at $A=B$ is not affected by the corrections. Notice that this last region $A=B$ is the one sharing

\footnotetext{
*lasma.alberte@gmail.com

†atteo.baggioli@uam.es, http://www.thegrumpyscientist.com

vcancer@ifae.es

§ujolas@ifae.es

Published by the American Physical Society under the terms of the Creative Commons Attribution 4.0 International license. Further distribution of this work must maintain attribution to the author(s) and the published articles title, journal citation, and DOI.
} 


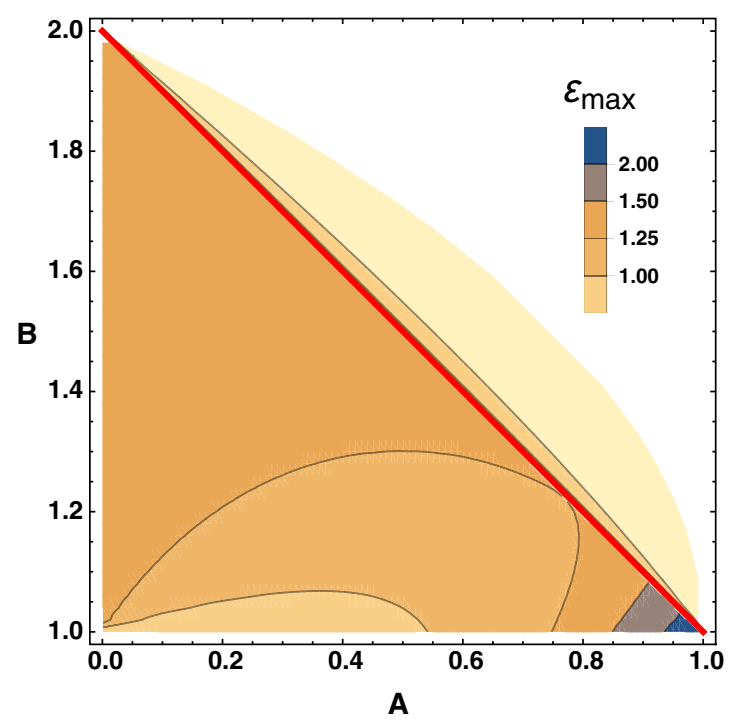

FIG. 1. The allowed parameter region for the potential $V(X, Z)=\rho_{\mathrm{eq}} X^{A} Z^{\frac{B-A}{2}}$. The left, bottom and right edges are respectively given by gradient instability, positivity of the bulk modulus, and superluminality. The red line separates the region where the maximum strain is due to the gradient instability (left) and the region where it is due to superluminality (right). Large strains are realized in the bluish area.

important similarities with the nonlinear response of rubber materials [2], as we explain in the Discussion section of [1]. In this sense, the match with the phenomenology of hyperelastic materials is better with these new results.

The full expression for $\varepsilon_{\max }$ as a function of $A, B$ is complicated but it simplifies in a certain region of the $A-B$ parameter space. Indeed, in the shaded area shown in Fig. 4, the form of $\varepsilon_{\max }$ is determined solely from avoidance of gradient instabilities $\left(c_{i}^{2}>0\right)$ and reduces to the simple analytical form

$$
\varepsilon_{\max }=\sqrt{2}\left(\frac{(B-1) B}{A-A^{2}-B+B^{2}}\right)^{1 / 4}
$$

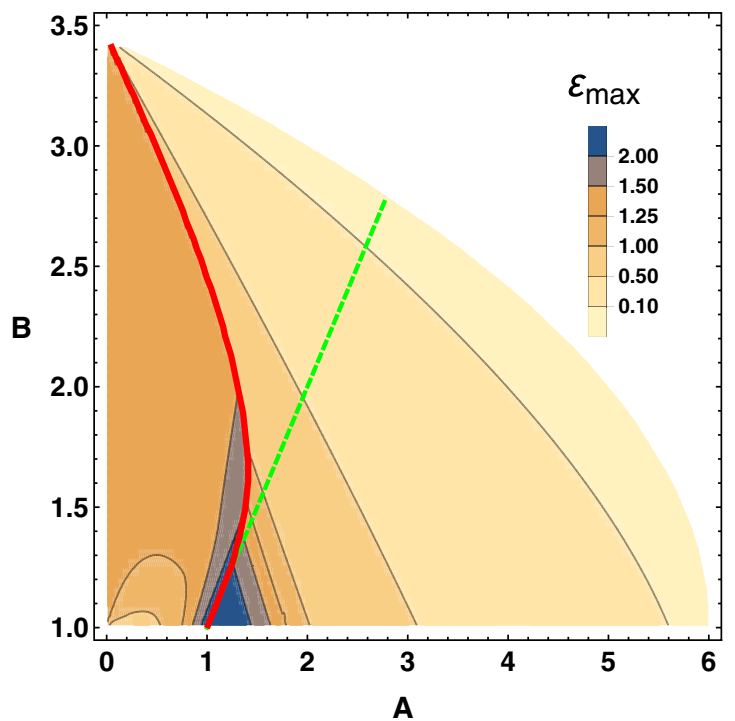

FIG. 2. The expanded parameter space for $v^{2}=0.2$. The red line splits the regions where the limit on the maximal strain comes from superluminality (on the right) and from gradient instability (on the left). The green dashed line is $A=B$. In the region $A \geq B$ the maximum strain is only dictated by subluminality. 


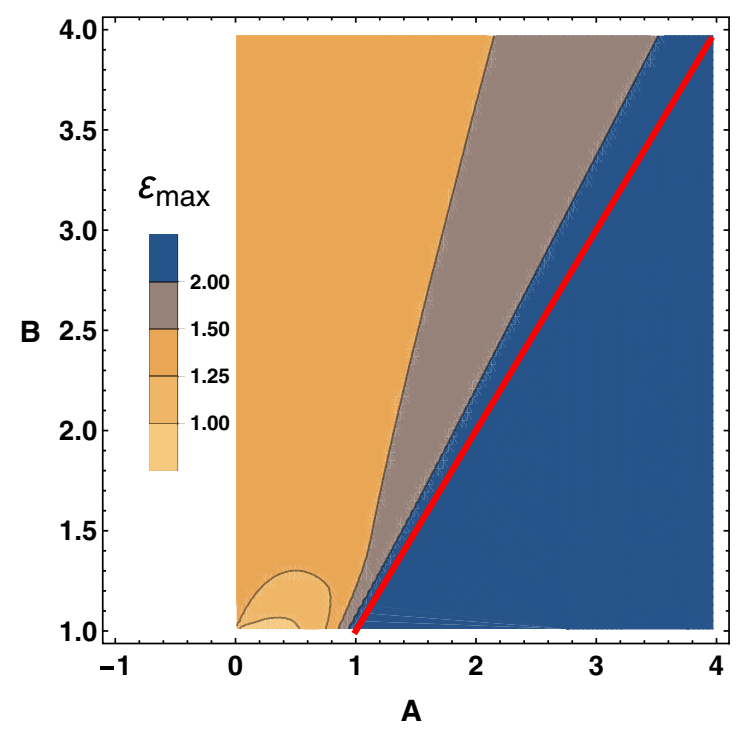

FIG. 3. The expanded parameter space for $v^{2}=10^{-8}$. The subluminal constraint in $A<B$ is now located at larger values of $A$ and $B$. In the region $A \geq B$ the maximum strain is only dictated by subluminality.

In [1] we had found that, in the limit $A \rightarrow 0, \varepsilon_{\max }$ increases asymptotically as

$$
\varepsilon_{\max } \simeq \sqrt{2}\left(\frac{B-1}{A}\right)^{1 / 4}
$$

While this is correct for the cases $\theta=0, \pi / 2$ it is not true that this $\varepsilon_{\max }$ is valid for any generic value of $\theta$, so our original result was not the most restrictive one. In particular, from Eq. (1), we observe that our previous interpretation of the above relationship (2) as a universal correlation between the maximum strain and exponents $A, B$ was not correct. Instead, taking into account this angle dependence, we find an interesting universal limit

$$
\varepsilon_{\max } \rightarrow \sqrt{2} \text { for } A \rightarrow 0
$$

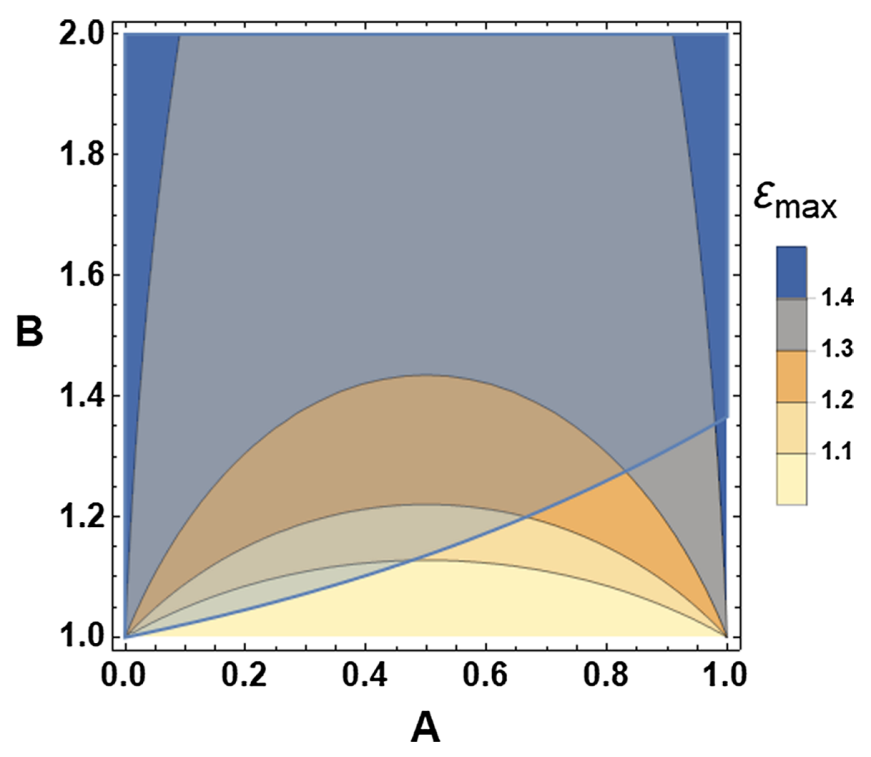

FIG. 4. Analytic solution for $\varepsilon_{\max }$ without imposing subluminality constraints. The simple expression (1) applies in the shaded area above the blue line. 


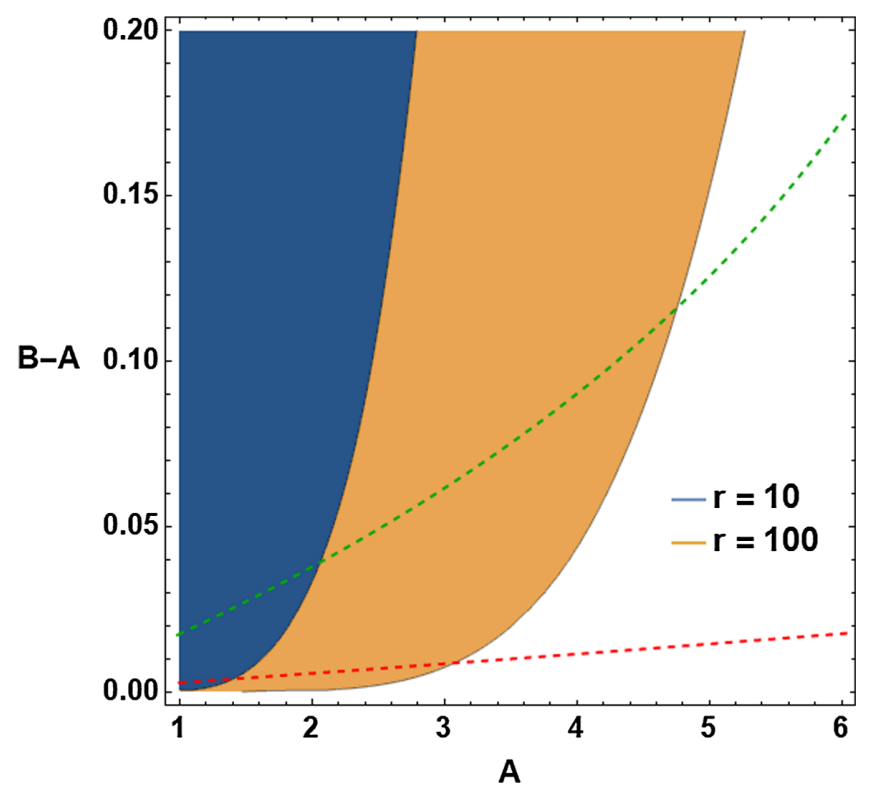

FIG. 5. Speed ratio constraints for $r=10,10^{2}$. Dashed lines show where $\varepsilon_{\max }=3,5$ (green, red).

Let us remark that the value for $\varepsilon_{\max }$ close to $A=0$ is found for an angle $\theta$ that obeys $\sin (2 \theta)=1 / \sqrt{3}$. For generic values of $A$ the value of $\theta$ leading to the most stringent condition on the maximal allowed strain has also been found analytically; however, its exact expression is too long to be given here.

In [1], we found another region where $\varepsilon_{\max }$ grows asymptotically, which is in the limit where $A \sim B$. This feature is still present, and it is given by the same expression up to a factor $2^{1 / 4}$ :

$$
\varepsilon_{\max } \sim\left(2 \frac{A}{B-A}\right)^{1 / 4}
$$

Finally we would like to revisit the ratio considered for the nonrelativistic potential between the speed $c_{+}$at zero strain and at the maximum shear deformation

$$
r \equiv \frac{c_{+}\left(\varepsilon_{\max }\right)}{c_{+}(\varepsilon=0)}
$$

which is shown in Fig. 5. Comparing with the one obtained originally we see that the parameter space has decreased only slightly. Thus, we continue to have a finite region where the $\varepsilon_{\max }$ is large and $r$ is moderately large (the material is nonrelativistic throughout the deformation). Interestingly, this includes values of the exponents $\nu_{\text {shear }}, \nu_{\text {bulk }}$ of order of a few-as seen in some real-world rubberlike materials [2].

[1] L. Alberte, M. Baggioli, V. C. Castillo, and O. Pujolas, Phys. Rev. D 100, 065015 (2019).

[2] Y. B. Fu and R. Ogden, Nonlinear Elasticity: Theory and Applications, London Mathematical Society Lecture Note Series Vol. 283 (Cambridge University Press, Cambridge, England, 2001). 